\title{
Thematic issue: Advanced modeling and innovative processes
}

\author{
Robertt Valente $^{1} \cdot$ Ricardo Alves de Sousa $^{1}$
}

Published online: 15 July 2015

(C) Springer-Verlag France 2015

The thematic issue on "Advanced Modeling and Innovative Processes" is a result of a kind invitation from the Editorial Board of the International Journal of Material Forming. Therefore, the Invited Editors would like to acknowledge the Editorial Board, in the person of the Editor-in-Chief Prof. Francisco Chinesta, the ESAFORM Association, in the person of its President Prof. Dorel Banabic and, last but not least, Springer Editions for this great opportunity.

The idea of this thematic issue is to give a summary (but comprehensive) overview of the most recent and diversified topics in the field of advanced modeling techniques, when directly applied to innovative processes. One of the main challenges of Engineering, in general aspects, is to provide a link between fundamental mathematical/physical concepts and industrial applications, leading to economic development as well as scientific one. This thematic issue is devoted to this landmark.

The contributions chosen to be included in the present thematic issue try to provide, as mentioned, a wide variety of examples where this link can be achieved. For instance, polymer crystallization phenomena are dealt with and simulated by means of numerical techniques including multi-scale effects. In other publication, also dealing with constitutive modeling, nonquadratic anisotropic criteria are considered for the correct simulation of metallic alloys. In order to achieve a

Robertt Valente

robertt@ua.pt

1 GRIDS Research Group, Department of Mechanical Engineering, University of Aveiro, Aveiro, Portugal higher level of efficiency in the numerical algorithms to be used in industrial applications, procedures for obtaining equivalent parameters from different yield criteria and hardening laws are studied and analyzed in another contribution. Directly related to industrial applications and constantly seeking for efficient, real-time computations, another contribution proposes innovative ways to choose optimum tool paths for incremental forming processes, based on sequencing and multi-step mesh morphing procedures. From the point of view of reliability and robustness of numerical simulation results, the thematic issue presents a paper dealing with an inevitable nonlinearity in forming processes: contact modeling, and its influence in results as coming from early stage decisions on the way to properly describe tools' surfaces. Also related to metal forming tools, another contribution deals with and presents a set of experimental setups able to properly investigate the wear of coatings, with results that can provide valuable insights into process simulation techniques, afterwards. Another analysis of coatings' wear, but this time applied to turning operations, is given in another contribution, widening the range of applications included in this thematic issue. Modeling and numerical analysis should also rely, when possible, on inverse techniques and optimization and a contribution is included in this issue dealing with an integrated approach for the SHPB test and ranging from numerical to experimental analysis. Another relevant field of study in the processes' modeling is the prediction of crack initiation and, subsequently, the monitoring of its growth. This topic is seen in a contribution dealing with FSW joining/processing techniques and the analysis of process parameters in the appearance of fracture zones and crack propagation. Finally, and also with the goal to widen the range of 
applications dealt with modeling/simulation techniques, a set of two contributions is included in the thematic issue, dealing with the study of ultrasonic compaction processes applied to composite laminates, one of them focusing on process modeling while a second one dedicated to the numerical simulation aspects of the analysis.
The Invited Editors hope this Thematic Issue can be a useful and relevant source of information on the challenging task of providing a better and more efficient way of communication between research and industry worlds, contributing to the essence of what Engineering must truly be.

Robertt Valente, Ricardo Alves de Sousa,

Invited Editors 